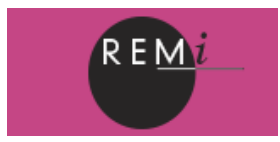

Revue européenne des migrations internationales

vol. 22 - n² | 2006

$20^{\mathrm{e}}$ anniversaire

\title{
Négociations identitaires marchandes
}

\section{Emmanuel Ma Mung}

\section{(2) OpenEdition}

\section{Journals}

Édition électronique

URL : https://journals.openedition.org/remi/2820

DOI : $10.4000 /$ remi. 2820

ISSN : $1777-5418$

Éditeur

Université de Poitiers

\section{Édition imprimée}

Date de publication : 1 juillet 2006

Pagination : 83-93

ISBN : 2-911627-42-3

ISSN : 0765-0752

Référence électronique

Emmanuel Ma Mung, « Négociations identitaires marchandes », Revue européenne des migrations internationales [En ligne], vol. 22 - n² | 2006, mis en ligne le 01 juin 2009, consulté le 15 avril 2022 URL : http://journals.openedition.org/remi/2820 ; DOI : https://doi.org/10.4000/remi.2820

Ce document a été généré automatiquement le 15 avril 2022.

(c) Université de Poitiers 


\title{
Négociations identitaires marchandes
}

\author{
Emmanuel Ma Mung
}

1 Le nombre d'entrepreneurs d'origine étrangère a considérablement augmenté durant les deux dernières décennies (Ma Mung et Dinh, 2007) mais le constat que nous faisions dans le précédent numéro anniversaire de la REMI $\left(1996\right.$, vol. 12, n ${ }^{\circ}$ ) sur le faible nombre de travaux leur étant consacré reste valable ${ }^{1}$ (Dinh, 2005). Numériquement faible si on la compare aux nombreuses publications concernant les questions migratoires (plusieurs milliers de références durant la dernière décennie), la recherche sur ce thème s'est néanmoins développée dans plusieurs directions fécondes. Dans ses travaux Anne Raulin s'est intéressée aux «centralités minoritaires » en privilégiant l'enjeu culturel que constituent la production et la consommation de biens culturels par et en direction des minorités (Raulin, 1996; Raulin, 2000). Une autre direction promue par Alain Tarrius dans les années quatre-vingt a donné lieu à la production la plus abondante. Les activités marchandes des populations migrantes y sont analysées, à partir d'un " paradigme de la mobilité » (Tarrius, 1989; Tarrius, 1989), comme une des manifestations des initiatives des migrants en relation avec le développement des circulations migratoires et la constitution de « territoires circulatoires » (Tarrius, 1995 ; Tarrius et Missaoui, 1995; Tarrius et Peraldi, 1995; Tarrius, 1996; Tarrius, 2001 ; Tarrius, 2002 ; Tarrius, 2005). Dans la même lignée des recherches se focalisent sur les circulations et les négociations des frontières, politiques et sociales (Hily et Rinaudo, 2002 ; Hily, 2005), les centralités commerçantes immigrées (Battegay, 2003), les places marchandes (Péraldi, 1999 ; Péraldi, 2001 ; Péraldi, 2001 ; Schmoll, 2004 ; Schmoll, 2005) ou portent sur la circulation de produits entre la France et le Maroc (Schaeffer, 2004). Une autre direction plus récente se situe dans une perspective historique et apparaît prometteuse (Bruno et Zalc, 2006). D'autres travaux se situant dans des perspectives différentes sont centrés sur des quartiers et/ou des populations particulières, chinoises (Auguin, 2004) ou indiennes (Jones, 2003). D'autres enfin mettent en relief la logistique mise en œuvre dans les échanges entre pays d'origine et d'installation (Tapia, 2002 ; Tapia, 2003). 
2 Dans un article paru dans le numéro de la REMI précité, j'avais abordé la question de la prévalence de l'appartenance ethnique dans le choix des partenaires économiques en développant deux idées: l'appartenance à un groupe ethnique constitue un cadre qui oriente de façon préférentielle, mais non exclusive, le choix des partenaires économiques, que ce soit dans ou hors de leur groupe respectif; le choix dans un groupe plutôt que dans un autre obéit à des mécanismes fondés sur la confiance, ellemême garantie par la réputation. Un thème connexe y était examiné mais de façon rapide, celui du lien entre l'entreprenariat ethnique et la négociation des identités, à partir de l'idée selon laquelle les entrepreneurs ethniques jouaient un rôle décisif dans cette négociation. L'on concluait que l'on était bien au cœur d'une négociation des identités puisque se nouait une transaction, s'établissait une manière d'accord à propos des valeurs des groupes, et s'élaboraient des présupposés partagés et des procédures d'implications réciproques qui confirmaient l'appartenance respective des acteurs à des groupes. Cette idée reposait sur l'intuition suivante: dans les transactions marchandes mettant en cause des partenaires spécifiés par leur appartenance à des groupes, se négocient, par le truchement des biens matériels échangés, des valeurs et des signes d'appartenance. C'est ce point qui sera développé ici en essayant de comprendre les mécanismes - ou plutôt les règles - qui y sont à l'œuvre. Cet aspect, à ma connaissance, n'a pas encore été abordé dans les travaux consacrés à l'entreprenariat ethnique.

\section{La présence paradoxale de l'étranger}

3 L'existence de populations d'origine étrangère sur le territoire national a pour effet d'engager des processus de négociation - le plus souvent symbolique - de la présence de ces populations (Ma Mung, 1994; Ma Mung, 1998; Ma Mung, 1998). Mais cette négociation concerne moins leur présence effective que les formes de cette présence. Ce n'est pas la présence de personnes d'origine étrangère en tant qu'individus séparés qui est en cause mais celle de groupes dont les membres se reconnaissent une origine commune, réelle ou putative, c'est-à-dire d'entités sociales qui affirment une identité de nature ethnique ${ }^{2}$ (ibid.). Parce qu'en France, reconnaître cette présence impliquerait à terme une reconnaissance institutionnelle qui entrerait en contradiction avec le modèle républicain d'incorporation à la nation, lequel - dans son principe - intègre des individus et non des groupes (Schnapper, 1994). Pourtant ces groupes existent de fait : ils se manifestent et sont produits comme différents de la société globale sur une base identitaire.

4 L'étranger a donc vocation à disparaître en tant que tel, à devenir un même (que nous). D'un autre côté, on recherche des produits et des services «étrangers » qu'il est seul susceptible de fournir. Autrement dit, l'étranger doit disparaître tout en restant présent. Il y a là comme un cercle qui a toutes les chances d'être vicieux puisqu'il se double d'un paradoxe : en désignant cet individu comme étranger on définit le groupe dans lequel on le range pour l'identifier comme tel tout en souhaitant que ce groupe disparaisse mais que l'individu, quant à lui, demeure. Mais il doit demeurer en tant qu'épicier " arabe ", restaurateur « chinois » etc. c'est-à-dire en tant que membre d'un collectif ethnique. Il doit exhiber des qualités (n'être pas n'importe quel épicier mais un épicier arabe, pas n'importe quel restaurateur mais un restaurateur chinois etc.) que par ailleurs il ne devrait pas manifester. L'entreprenariat ethnique expose ces 
situations de manière exemplaire ${ }^{3}$. C'est au point de retournement de ce paradoxe que la négociation des identités se situe, et c'est ce cercle qu'elle tente d'ouvrir par l'institution d'une communauté de circonstance - la communauté marchande comme nous allons essayer de le voir.

\section{Une négociation symbolique}

5 Cette négociation des identités est symbolique à plusieurs titres. D'une part, elle n'est pas posée explicitement comme telle par les protagonistes. Ensuite, elle concerne les attributs des identités qui en constituent en quelque sorte les signifiants. Enfin, la transaction marchande elle-même porte sur des « objets » constitués par les produits et services, et non sur les identités elles-mêmes. Dans la transaction marchande s'en réalise une autre. La première est le moyen d'une seconde dans laquelle se négocient, à travers les « objets " échangés, les représentations réciproques des groupes auxquels appartiennent les échangistes, lesquelles affectent les identités collectives.

6 Comme les objets échangés sont le support de cette transaction, une signification spéciale leur est attachée : ils représentent quelque chose du groupe auquel appartient chacun des échangistes. C'est-à-dire qu'en termes plus généraux, ils possèdent des propriétés qui définissent chacun des sujets de l'échange. Et le même objet permet de définir l'identité de deux sujets différents.

1. Ainsi cet objet de consommation pourtant courante n'est pas n'importe quel objet: ce paquet de lessive acheté chez l'épicier arabe de mon quartier n'est pas celui, pourtant identique, acheté dans une grande surface : il est celui que m'a vendu ce commerçant que je sais appartenir à un groupe tel que je sais que je trouverai chez lui ce produit à l'angle de la rue, dimanche à 21 heures. Ce produit est caractérisé, qualifié, par une relation sociale particulière : celle d'un acheteur qui, à travers la représentation qu'il se fait d'un vendeur, assimile ce dernier à un groupe dont il sait que les membres commerçants vont offrir un service spécial (être ouvert dimanche à 21 heures). L'important ici est l'assimilation à un groupe social, le fait qu'à travers le produit et dans l'acte d'échange, l'on confère une identité sociale à un individu.

2. Il y a une caractérisation ethnique du commerçant de la part du client mais il y en a une symétrique de la part du commerçant à propos du client qui range ce dernier dans un groupe. Il n'y a là rien que de très banal dans l'entreprenariat ethnique. Par exemple, les commerçants chinois qui approvisionnent les populations africaines distribuent les produits qu'ils savent être ceux recherchés par ces populations. Ils les disposent dans leur magasin et les conditionnent de la façon dont ils pensent qu'elles souhaitent les trouver. Il en va de même pour les commerces orientés vers les populations autochtones ou identifiées comme telles par les tenanciers, ou encore pour les magasins tournés vers les populations asiatiques. Pour l'épicier arabe de quartier, c'est une offre de produits et surtout de services qu'il pense être ceux recherchés par un certain type de clientèle : l'autochtone par exemple. Le commerçant ainsi identifie ethniquement sa clientèle ${ }^{4}$. Les deux sujets définis par l'objet (le paquet de lessive) sont, dans cet exemple, l'épicier « arabe » et le client « autochtone », il est facile d'imaginer bien d'autres situations.

7 Les propriétés des objets qui définissent les sujets leur viennent d'une relation sociale dans laquelle se marque l'appartenance des individus à un groupe. Les gestes (achat, vente) à travers lesquels s'expriment les statuts sociaux des échangistes (acheteur autochtone, vendeur arabe) donnent à ces objets des significations extrinsèques (objet vendu par un commerçant arabe, objet acheté par un autochtone) qui se superposent à 
leurs caractères intrinsèques (poudre à laver). C'est comme s'il y avait dans l'objet quelque chose de plus que sa valeur d'usage qui lui est ordinairement attachée lorsqu'on l'achète dans un magasin "anonyme " (un paquet de lessive pour laver mon linge). C'est que le même objet se présente différemment en fonction de la relation sociale dans laquelle il apparait.

Quelle est cette relation sociale ? Quelle est cette différence ? Cette relation, dans le cas de l'économie ethnique, est un échange qui intervient entre deux individus qui s'attribuent réciproquement des identités sociales. On pourrait dire la même chose de n'importe quel acte d'achat dans un commerce quel qu'il soit: au minimum, l'un des protagonistes est identifié comme acheteur, l'autre comme vendeur. La différence, ici, tient en ce que cette identité sociale est une identité ethnique qui suppose que chacun repère l'origine de l'autre. Le statut social des échangistes n'est pas seulement celui d'un distributeur ou d'un consommateur, il est défini par le fait que chacun range l'autre dans un groupe dont il suppose que les membres partagent une origine commune. Il y a donc quelque chose d'étonnant qui se passe: d'une part une distinction, une séparation qui est au fondement de l'économie ethnique (la prévalence de l'appartenance ethnique dans le choix des partenaires économiques, à comprendre comme fonctionnant dans les relations intra-groupe et intergroupe - exemple ici développé), et d'autre part, la manifestation d'appartenance à une même communauté (au moins celle constituée par les individus qui tombent d'accord pour acheter et vendre), la non-appartenance de l'un ou de l'autre à la communauté des échangistes interdisant l'échange.

Qu'y a-t-il d'autre dans l'objet que sa valeur d'usage ordinaire? Porte-t-il des signes identitaires? Si cela est évident, de prime abord, pour certains objets exotiques (couscous ou nems... pour prendre des exemples stéréotypés ${ }^{5}$ ), cela l'est moins pour les objets du commerce courant. Un paquet de lessive acheté chez un épicier arabe a-t-il une autre signification que celle d'un produit de lavage? Si oui, quelle signification de plus lui attribuons-nous? (Quelle propriété a-t-il, en plus, qui définisse les sujets?) Comme on l'a dit, il est l'argument d'une relation sociale qui met en cause le statut social (identitaire) à travers des gestes qui définissent les sujets en leur attribuant une identité. Le supplément que l'on trouve donc est son utilité comme support d'opérations d'identification. La propriété supplémentaire que nous lui attribuons est celle de définir à travers l'échange nos identités respectives, de permettre et d'être le support d'un échange identitaire. La signification que nous lui attribuons est qu'il est possible d'échanger avec un individu différent et ce faisant, de constituer une même communauté.

\section{Une double réduction de l'identité}

10 Avec la description que nous venons de faire, nous nous trouvons devant une configuration paradoxale qui se présente ainsi :

1. d'une part, il y a identification respective (quelque chose comme une séparation) et d'autre part, il y a appropriation (quelque chose comme une fusion). Ou dit autrement :

2. il y a d'une part, un rapport social qui confirmerait l'appartenance des échangistes à des groupes distincts, et d'autre part, une appropriation de symboles qui modifierait l'appartenance des individus. Lorsque l'autochtone va chez l'épicier arabe il s'approprie des attributs de son identité puisqu'il achète, en plus du produit (poudre à laver), le service 
spécial qui lui est attaché (être ouvert le dimanche etc.), et réciproquement pour l'épicier puisqu'il se conforme aux usages de consommation de son client. Tout comme lorsque l'autochtone mange un couscous chez un restaurateur tunisien (ou marocain), il s'approprie en plus du produit (semoule de blé, légumes et viande) des attributs de son identité (le fait qu'il s'agit d'un couscous et non de semoule). Et réciproquement de la part du restaurateur puisqu'il va apprêter son produit en fonction des attentes de son client. Ce paradoxe, cette séparation-fusion, est probablement constitutif de la relation sociale. C'est à condition de définir nos identités respectives (se définir soi-même et définir l'autre) que nous pouvons entrer en relation, sinon il y a indistinction, fusion totale et donc absence de relations ${ }^{6}$. Mais il faut aussi trouver à se rejoindre sinon il y a séparation totale et, là aussi, absence de relations.

11 Dans ces moments de l'échange se constitue une communauté à travers l'échange d'attributs identitaires, donc d'altérités réciproques. Comment des altérités peuventelles êtres "échangées ", être rendues équi-valentes (de même valeur) puisque par définition elles sont extérieures les unes aux autres? Comment l'altérité peut-elle être convertie en identité ?

L'échange est possible parce qu'il y a une double réduction :

1. une réduction à l'identique (que l'on pourrait appeler en jouant un peu des mots « réduction identitaire ») par le biais de l'échange marchand, selon la thèse classique de la réduction de la qualité en quantité, de la valeur d'usage en valeur d'échange par le biais d'un équivalent général. Ainsi, l'altérité des sujets est transformée en une simple différence quantitative, en une équivalence : une variation de l'identité ;

2. une réduction objective, ou objectivante, du fait que l'échange concerne des objets.

13 Cette double réduction permet la désaffectation-réaffectation des attributs identitaires : ceux-ci, d'une part changent de sujet et, d'autre part, ne sont plus chargés des affects qui leur sont habituellement attachés. La première réduction a pour effet que les objets sont échangeables : réduction marchande à l'identique. La seconde a pour conséquence que bien qu'ils soient les supports ou les symboles d'un rapport social dans lequel se définit l'appartenance des échangistes, en tant qu' «objets ", ils ont quelque chose d'extérieur à cette relation, une extériorité qui permet l'échange. Cette double réduction permet non seulement l'échange matériel, mais aussi l'échange de symboles identitaires. Grâce à elle, les signes de l'appartenance ethnique perdent de leur puissance affective : bien que les attributs identitaires soient souvent conçus par les sujets comme non échangeables parce que constitutifs de l'identité, ils sont, de fait, échangés dans l'acte marchand ${ }^{7}$. Se réalise ainsi un échange de quelque chose qui, de prime abord, peut apparaître comme inaliénable, intrinsèque à l'identité des sujets et donc non négociable. C'est que l'identité dont il est question est une identité relative - une identité définie par des attributs : des qualités (ou des défauts), des prédicats que l'on attribue à des personnes - et non une identité absolue qui dans le cas des identités sociales n'aurait aucun sens. C'est dire que l'échange marchand, à travers la réduction à l'identique, "désaffecte " de leurs affects les identités et en permet la négociation. L'extériorité de ces objets s'en trouve en quelque sorte renforcée, elle a pour effet qu'ils s'imposent à nous à la manière des objets " matériels » dont on ne peut mettre en cause la présence $d u$ fait même de leur caractère objectif. Il y a donc quelque chose de contraignant dans ces objets, un peu à la façon dont les choses "naturelles " s'imposent à nous. De sorte que par leur truchement ce sont des usages de ces objets que nous nous imposons : des règles de la vie quotidienne. La contrainte n'est donc pas naturelle mais sociale. Elle est une façon commune d'agir et de penser. Une façon de faire avec les 
objets qui est une manière d'être avec les individus exprimant un sens que nous avons introduit dans notre vie quotidienne. Ce sens, confirmé au jour le jour, est qu'il est possible de commercer, d'échanger avec l'autre. La dimension marchande de l'échange (la réduction sur le prix à payer contenue dans l'objectivation marchande) permet la négociation des identités parce qu'elle révèle la dimension relative de l'identité sociale du fait même que l'échange porte sur des attributs (et non sur les identités elles-mêmes puisque celles-ci n'existent pas dans l'absolu). Qu'il y ait échange et négociation signifie également qu'il y a apaisement, recherche de résolution d'un conflit potentiel, évitement d'un affrontement possible, pacification. Et le commerce permet, selon la formule célèbre, d'échanger des biens plutôt que des coups. Les opérations qui permettent, à notre époque et dans notre société, à des populations différentes de cohabiter sont celles de l'échange marchand (quantification réductrice et réduction identitaire) parce que s'y manifeste le caractère relatif des identités ethniques et que s'y élaborent des règles que les échangistes introduisent dans leur vie quotidienne.

\section{Conclusion}

L'entreprenariat ethnique comme phénomène tangible nous invite à reconsidérer certaines formes d'échange et les extensions spatiales qui leur sont liées (quartiers commerçants "ethniques ", places marchandes cosmopolites...) à partir d'un point de vue identitaire puisque c'est celui à partir duquel les acteurs nous disent agir (tout le vocabulaire qu'ils utilisent est empreint des stéréotypes les plus forts). Il est aussi celui à partir duquel nous arrivons, paradoxalement, à penser qu'il ne nous permet pas d'appréhender adéquatement le réel puisque pour l'avaliser il faudrait que l'identité de la réalité à ce que nous en disons soit parfaite, et il y a là comme un trouble à propos de l'identité de l'identité. Il est enfin celui qui nous laisse voir que dans un cas où la dimension identitaire se manifeste de la façon la plus forte (l'entreprenariat ethnique) celle-ci n'est pas séparable de la recherche de l'altérité puisque ce qui relie les individus engagés dans ces transactions n'est pas tant la recherche du proche et du Même comme garant de la confiance indispensable à leur bon déroulement que celle de l'Autre comme assurance contre une fusion qui interdirait l'échange en abolissant toute distance. De sorte que le point de vue identitaire se renverse et appelle son contraire. Ce que nous avons observé à l'échelle microscopique se déploie également à de très vastes échelles car c'est sur les mêmes usages et règles que s'établissent les relations entre partenaires très distants. L'étude des transactions marchandes qui se réalisent sur une base identitaire nous permet de circonscrire cette zone incertaine où se réalise l'échange, dans un équilibre précaire et toujours remis en cause entre des proximités et des distances autant identitaires/ altéritaires que spatiales. À ce titre elle demande à être développée par exemple dans la perspective de la "remontée des Sud " que dessine Alain Tarrius dans le présent numéro. 


\section{BIBLIOGRAPHIE}

AUGUIN Estelle (2004) Le nouvel an chinois à Paris, théâtre d'économie ethniques, Revue Européenne des Migrations Internationales, vol. 20, n 3, pp. 75-87.

BATTEGAY Alain (2003) Les recompositions d'une centrale commerçante immigrée : La Place du Pont à Lyon, Revue Européenne des Migrations Internationales, vol. 19, n², pp. 9-22.

BRUNO Anne-Sophie, ZALC Claire (2006). Petites entreprises et petits entrepreneurs étrangers en France (XIX-XX ${ }^{e}$ siècle). Paris, Actes de l'histoire de l'immigration, Paris, Ed. Publibook Université, 275 p.

DINH Bernard (2005) L'entreprenariat ethnique en France et dans le monde anglo-saxon, Bilan des connaissances, inventaire bibliographique commenté, Rapport de recherche pour le FASILD, Paris, $130 \mathrm{p}$.

ENDELSTEIN Lucine (2006) Les Juifs originaires d'Afrique du Nord acteurs du développement du commerce casher, in Zalc Claire Bruno Anne-Sophie (ed.) Petites entreprises et petits entrepreneurs étrangers en France (XIX ${ }^{e}-\mathrm{XX}^{e}$ siècle), Paris, Ed. Publibook Université. pp. 181-193.

HILY Marie-Antoinette (2005) Circuler et commercer à la frontière : Vintimille/Menton, in Stéphane de Tapia, La création d'entreprises par les immigrés, un dynamisme venu d'ailleurs, Paris, L'Harmattan, pp. 137-170.

HILY Marie-Antoinette, RINAUDO Chirstian (2002) Vintimille, ville-frontière, ville-marché : l'espace des circulations et du commerce, in Marie-Antoinette Hily et Emmanuel Ma Mung, Catégories et lieux des circulations migratoires. Rapport de recherche pour la Mission Recherche, convention $\mathrm{n}^{\circ}$ WKO 324, Paris, Ministère du travail, pp. 48-62.

JONES Graham (2003) Le trésor caché du quartier indien : esquisse ethnographique d'une centralité minoritaire parisienne, Revue Européenne des Migrations Internationales, vol. 19, $\mathrm{n}^{\circ} 1$, pp. 233-243.

MA MUNG Emmanuel (1994) L'entreprenariat ethnique en France, Sociologie du travail, $\mathrm{n}^{\circ} 2$, pp. 185-209.

MA MUNG Emmanuel (1996) Entreprise économique et appartenance ethnique, Revue Européenne des Migrations Internationales, vol. 12, $\mathrm{n}^{\circ}$ 2, pp. 211-233.

MA MUNG Emmanuel (1998) Ethnic economy and diaspora, in Shiji zhijiao haiwai huaren [ethnic chinese at the turn of century], al. Xiamen, Fujian Renmin Chubanshe, pp. 26-60.

MA MUNG Emmanuel (1998) Territorialisation marchande et négociations des identités, les Chinois à Paris, Espaces et Sociétés, $\mathrm{n}^{\circ} 4$.

MA MUNG Emmanuel (1999) Autonomie, migrations et altérité, Université de Poitiers, 3 vol. (HDR : Géographie : Poitiers : Univ. Poitiers : 1999)

MA MUNG Emmanuel, DINH Bernard (2007) (à paraître) The new landscapte of ethnic entrepreneurship in France, in L. Dana, Ethnic entrepreneurship in Europe, Routledge ed.

MULLER Laurent, de TAPIA Stéphane, (2005) La création d'entreprises par les immigrés, un dynamisme venu d'ailleurs, Paris,L'Harmattan, $270 \mathrm{p}$.

PERALDI Michel (1999) Marseille : réseaux migrants transfrontaliers, place marchande et économie de bazar, Cultures \& Conflits, $\mathrm{n}^{\circ}$ 33-34, pp. 51-67. 
PERALDI Michel (2001) Cabas et containers : activités marchandes informelles et réseaux migrants transfrontaliers, Paris, Maisonneuve et Larose : 361 . (Frontières, villes : lieux de passage)

PERALDI Michel (2001) L'esprit de bazar : mobilités transnationales maghrébines et sociétés métropolitaines. Le comptoir démantelé, in Michel Peraldi, Cabas et containers : activités marchandes informelles et réseaux migrants transfrontaliers, Paris, Maisonneuve et Larose, pp. 32-64.

RAULIN Anne (1996) Stratégies identitaires et négociations inter-culturelles : des diasporas commerçantes à Paris, in Nicole Haumont, La ville, agrégation et ségrégation sociales, Paris, L'Harmattan, pp. 73-83.

RAULIN, Anne (2000) L'ethnique est quotidien : diasporas, marchés et cultures métropolitaines, Paris, L'Harmattan, 229 p. (Connaissance des hommes)

SCHAEFFER Fanny (2004) Le territoire des passe-frontières, dynamiques sociales, identitaires et spatiales de la structuration du champ migratoire marocain, Université de Poitiers, 478 p. (Thèse : Géographie : Poitiers : Univ. Poitiers : 2004)

SCHMOLL Camille (2004) Une place marchande cosmopolite, Dynamiques migratoires et circulations commerciales à Naples, Université Paris 10,550 p. (Thèse : Sociologie : Nanterre : Univ. Paris 10 : 2004)

SCHMOLL Camille (2005) Pratiques spatiales transnationales et stratégies de mobilité des commerçantes tunisiennes, Revue Européenne des Migrations Internationales, vol. 21, n 1 , pp. 131-154.

SCHNAPPER Dominique (1994) La communauté des citoyens : sur l'idée moderne de nation, Paris, Gallimard, 228 p.

SIMMEL Georg (1986) Digression sur le problème : Comment la société est-elle possible ?, in p. Watier, Georg Simmel, la sociologie et l'expérience du monde moderne, Paris, Méridiens Klincksieck, pp. 21-46.

TAPIA Stéphane de (2002) Migration transnationale et entrepreneuriat migrant : le cas turc, in Jean-Luc Richard, Immigration, marché du travail, intégration, Paris, La Documentation Française (coll. des Rapports Officiels), pp. 167-178.

TAPIA Stéphane de (2003) Transnational Migration and Entrepreneurship of Migrants : Between Turkey, Europe and Turkic World, in Danielle Juteau, The Social Construction of Diversity (Recasting the Master of Industrial Nations), New York, Berghahn Books Publ., pp.65-82.

TARRIUS Alain (1989) Perspectives phénoménologiques dans l'étude de la mobilité, in Transports et sciences sociales, questions de méthode, Caen, Paradigme, pp. 47-81.

TARRIUS Alain (1989) Anthropologie du Mouvement, Caen, Ed. Paradigme, 185 p.

TARRIUS Alain (1995) Naissance d'une colonie : un comptoir commercial à Marseille, Revue Européenne des Migrations Internationales, vol. 11, n 1, pp. 21-52.

TARRIUS Alain (1996) Territoires circulatoires et espaces urbains, in Mirjana Morokvasic, Migrants : les nouvelles mobilités en Europe, Paris, L'Harmattan, pp. 93-117.

TARRIUS Alain (2001) Au-delà des États-nations : des sociétés de migrants, Revue Européenne des Migrations Internationales, vol. 17, $\mathrm{n}^{\circ} 2$, pp. 37-62.

TARRIUS Alain (2002) La mondialisation par le bas, les nouveaux nomades de l'économie souterraine, Paris, Balland, 168 p. 
TARRIUS Alain (2005) Le lien fort comme préalable à la réussite économique, in Stéphane de Tapia, La création d'entreprises par les immigrés, un dynamisme venu d'ailleurs, Paris, L'Harmattan, pp. 21-51.

TARRIUS Alain, MISSAOUI Lamia (1995) Arabes de France dans l'économie mondiale souterraine, Paris, Ed. de l'Aube, 219 p. (Essai)

TARRIUS Alain, PERALDI Michel (dir.) (1995) Marseille et ses étrangers, Revue Européenne des Migrations Internationales, vol. 11, n 1, $219 \mathrm{p}$.

WEBER Max (1971) Economie et société, Paris, Plon.

\section{NOTES}

1. À noter toutefois la parution récente d'un ouvrage dirigé par Laurent Muller et Stéphane de Tapia (Muller et De Tapia, 2005) regroupant une dizaine de contributions sur le sujet.

2. On retient ici la définition wéberienne de l'identité ethnique (Weber, M. (1971) Economie et société, Paris, Plon); pour lequel ce qui différencie l'identité ethnique des autres identités collectives (religieuse, professionnelle...) est la croyance partagée dans une origine commune, réelle ou supposée. On peut remarquer, comme cela a dû être déjà fait, que la définition de Weber est opératoire (utilisable et applicable à différentes populations) mais pas «statistique »: les catégories ethniques ainsi définies ne sont ni exhaustives (on ne peut y ranger tous les individus), ni exclusives (dans certaines circonstances, un individu peut appartenir à plusieurs catégories). On ne peut donc comptabiliser des individus sur cette base-là que de façon approximative.

3. L'expression entreprenariat ethnique désigne ici les entreprises commerciales ou artisanales dans lesquelles les relations entre partenaires économiques s'établissent sur la base de leur appartenance à un groupe ethnique. Il est important de souligner trois choses: (1) cette appartenance n'est pas le seul motif de ces relations car y interviennent bien entendu des considérations qui n'ont rien à voir avec cette appartenance : qualité du produit ou du service, solvabilité de l'acheteur, caractéristiques personnelles du vendeur etc. (2) cette appartenance a des effets sur les relations entre agents du même groupe (exemple du commerce communautaire) mais aussi entre individus appartenant à des groupes différents (exemple du commerce exotique ou du commerce courant) (3) l'entreprenariat ethnique au sens qui lui est donné ici ne désigne pas seulement les entreprises orientées vers le groupe dont elles sont issues (type communautaire) mais aussi les autres (type exotique ou type banal).

4. Cette définition réciproque intervient d'ailleurs dans d'autres domaines de l'activité économique tels que la confection par exemple dans le Sentier où les protagonistes sont identifiés comme donneurs d'ordre juifs, façonniers chinois ou turcs, porteurs cingalais (Ma Mung, E. (1996). Entreprise économique et appartenance ethnique. Revue européenne des migrations internationales, vol. 12, pp. 211-233).La caractérisation ethnique n'est donc pas le fait d'un seul des échangistes mais des deux.

5. Cela permet de noter en passant que même les objets « exotiques " ne contiennent pas, par essence, de marque ethnique : un nem est une farce enveloppée dans une feuille à base de farine de riz (ou de blé), il ne devient nem que lorsque les individus se le représentent comme nourriture habituellement consommée par les populations asiatiques.

6. C'est dire ainsi que l'altérité est au fondement de la relation sociale. Nous retrouvons ainsi une interprétation que l'on peut faire d'un texte de George Simmel (Simmel, 1986 : 21-46) Voir aussi Ma Mung, 1999.

7. Une bonne illustration des effets de ce genre de réduction est donnée par les commerces casher étudiés par Lucine Endelstein (2006). Elle montre que certains commerçants ouvrent des 
établissements casher de très stricte surveillance alors qu'ils sont eux-mêmes peu pratiquants. Ils expliquent que cela leur permet de toucher une clientèle plus large (la clientèle habituelle plus les pratiquants et les très pratiquants).

\section{RÉSUMÉS}

Dans les transactions marchandes mettant en cause des partenaires spécifiés par leur appartenance à des groupes, se négocient, par le truchement des biens matériels échangés, des valeurs et des signes d'appartenance. Cet article essaie de comprendre les mécanismes - ou plutôt les règles - qui sont à l'œuvre dans cet échange. Celui-ci se réalise à travers une double réduction identitaire (une réduction à l'identique et une réduction objective) dans laquelle les objets troqués perdent leurs attributs identitaires particuliers et les affects qui leur sont liés pour devenir des choses échangeables. L'extériorité de ces objets s'en trouve renforcée, de sorte qu'ils s'imposent à nous. Mais de cette manière c'est aussi les usages de ces objets que nous nous prescrivons, des façons de faire et d'être avec les gens: des règles de la vie quotidienne qui expriment un sens que nous y avons introduit. Celui qu'il est possible d'échanger toutes choses avec l'autre.

Identity transactions in business exchanges. In businesses involving partners specified through the groups to which they belong, values and marks of belonging are mediated by material goods exchanged. The present paper tries to understand the mechanisms - or rather the rules - at work in that type of exchange. It is operated through a double reduction of identity (reduction to similarities and objective reduction), in which the objects exchanged loose their specific identity attributes and the affects linked to them in order to become tradable goods. The exteriority of these objects is thereby reinforced, so that they impose themselves upon us. But in so doing, they also prescribe the use which is made of them, as well as ways of being and of behaving with people: they are rules for daily life which express the meaning we have put into them. That means that we can exchange everything with other people.

Negociaciones de identidad comerciales. En aquellas transacciones comerciales que ligan a socios « especificados" por su pertenencia a grupos se negocian valores y signos de pertenencia mediante el intercambio de bienes materiales. Este artículo trata de comprender los mecanismos, o más bien las reglas, que participan en este intercambio. Este último se realiza a través de una doble reducción de identidad (reducción idéntica y reducción objetiva) en la cual los objetos intercambiados pierden sus atributos de identidad particulares y los afectos que conllevan para convertirse en cosas intercambiables. La exterioridad de estos objetos se ve reforzada de tal manera que los objetos se nos imponen y, con ellos, los usos que nos prescribimos de dichos objetos. Maneras de hacer y de comportarse con la gente; reglas de la vida cotidiana que expresan un sentido que nosotros mismos hemos introducido: el de que es posible intercambiar cualquier cosa con el otro.

\section{INDEX}

Mots-clés : appartenance, commerce, identité ethnique, relations interculturelles 


\section{AUTEUR}

\section{EMMANUEL MA MUNG}

Géographe, Directeur de recherche au CNRS, Migrinter (Unité mixte de recherche 6588 CNRS), Maison des Sciences de l'Homme et de la Société, 99 av du Recteur Pineau, Poitiers, France.

Courriel : emmanuel.mamung@univ-poitiers.fr 